Research Article

Majid Abbasov* and Faramoz Aliev

\title{
Modifications of the Charged Balls Method
}

https://doi.org/10.1515/comp-2020-0008

Received Jul 09, 2019; accepted Dec 15, 2019

Abstract: The Charged Balls Method is based on physical ideas. It allows one to solve problem of finding the minimum distance from a point to a convex closed set with a smooth boundary, finding the minimum distance between two such sets and other problems of computational geometry. This paper proposes several new quick modifications of the method. These modifications are compared with the original Charged Ball Method as well as other optimization methods on a large number of randomly generated model problems.

We consider the problem of orthogonal projection of the origin onto an ellipsoid. The main aim is to illustrate the results of numerical experiments of Charged Balls Method and its modifications in comparison with other classical and special methods for the studied problem.

Keywords: charged balls method, computational geometry, optimization, mathematical programming

\section{The problem statement and the initial algorithm}

Consider the problem

$$
\left\{\begin{array}{l}
\|x\| \longrightarrow \min , \\
x \in X .
\end{array}\right.
$$

of orthogonal projection of the origin onto a set

$$
X=\left\{x \in \mathbb{R}^{n} \mid f(x) \leq 0\right\},
$$

where $f: \mathbb{R}^{n} \rightarrow \mathbb{R}$ is a convex twice continuously differentiable function and $\mathbf{0} \notin X$. We can assume that $\nabla f(x) \neq \mathbf{0}$ for all $x \in \mathrm{bd} X$, where $\mathrm{bd} X$ is the boundary of the set $X$.

*Corresponding Author: Majid Abbasov: St. Petersburg State University, SPbSU, 7/9 Universitetskaya nab., St. Petersburg, 199034 Russia; Email: m.abbasov@spbu.ru

Faramoz Aliev: St. Petersburg State University, SPbSU, 7/9 Universitetskaya nab., St. Petersburg, 199034 Russia; Email: faramoz.aliev@gmail.com
The continuous version of the Charged Ball Method has a form [1]:

$$
\left\{\begin{array}{l}
\dot{x}=z, \\
\dot{z}=p_{1} \psi(x)-p_{2} z-\chi(x, z),
\end{array}\right.
$$

where

$$
\begin{gathered}
\psi(x)=-\frac{1}{\|x\|^{3}} x+\frac{\langle x, \nabla f(x)\rangle}{\|x\|^{3}\|\nabla f(x)\|^{2}} \nabla f(x), \\
\chi(x, z)=\frac{\langle H(x) z, z\rangle}{\|\nabla f(x)\|^{2}} \nabla f(x),
\end{gathered}
$$

$\nabla f(x)$ and $H(x)$ are the gradient and the Hessian matrix of the function $f$ at the point $x$, and $p_{1}, p_{2}$ are parameters.

Applying Euler's method for the solution of system (2) we get an iterative algorithm for the problem. Let us describe it.

Choose some small $\delta>0, \varepsilon>0$. Let $x_{0}$ and $z_{0} \neq \mathbf{0}$ be given.

1. Build

$$
\left\{\begin{array}{l}
x_{k}=x_{k-1}+\delta z_{k-1}, \\
z_{k}=z_{k-1}+\delta\left(p_{1} \psi\left(x_{k-1}\right)-p_{2} z_{k-1}\right. \\
\left.-\chi\left(x_{k-1}, z_{k-1}\right)\right) .
\end{array}\right.
$$

2. Check a stopping criteria

$$
\left\|\psi\left(x_{k}\right)\right\|<\varepsilon .
$$

- If it is fulfilled, we take $x_{k}$ as the solution and exit the algorithm.

- If it is not, then increment $k$ by one and return to the first step.

\section{Modifications of the method}

One of the essential features of the Charged Balls Method (3) is that the position of a point $x_{k}$ varies slightly at initial iterations. In addition the point $x_{k}$ passes through the solution and make damping oscillations in its neighborhood.

Both of these effects are associated with the inertia which is brought by the presence of mass (see. [1]). To overcome these effects, several modifications of the Charged Ball Method are proposed. 


\subsection{Zero-mass algorithm}

Assuming that the mass in the differential equation of motion (2) equals to zero, we obtain the system

$$
\left\{\begin{array}{l}
\dot{x}=z, \\
0=p_{1} \psi(x)-p_{2} z-\chi(x, z) .
\end{array}\right.
$$

To pass from the continuous method (5) to its discrete variant we can linearize the second equation of the resulting system in order to recalculate the variable $z_{k}$ at each iteration. Denote

$$
\Phi(z)=p_{1} \psi(x)-p_{2} z-\chi(x, z) .
$$

Let $z_{k}$ be given. We can find the next point $z_{k+1}$ from the relation

$$
\Phi\left(z_{k}\right)+\Phi^{\prime}\left(z_{k}\right)\left(z_{k+1}-z_{k}\right)=0,
$$

where

$$
\Phi^{\prime}\left(z_{k}\right)=-p_{2} E-2 \frac{\nabla f(x) z^{T} H\left(x_{k}\right)}{\left\|\nabla f\left(x_{k}\right)\right\|^{2}},
$$

and $E$ is the identity matrix. Therefore choosing a small $\delta>0$ as a step size we get the algorithm

$$
\left\{\begin{array}{l}
x_{k+1}=x_{k}+\delta z_{k}, \\
z_{k+1}=z_{k}-\left[\Phi\left(z_{k}\right)\right]^{-1} \Phi\left(z_{k}\right) .
\end{array}\right.
$$

\subsection{Algorithm with speed zeroing}

Another way to deal with the effects associated with the presence of mass is to zero the initial velocity at each iteration. This leads to the movement along the direction tangential to the boundary of the set. Hence a correction procedure (local projection) is required to provide that the point belongs to the boundary of the set.

Describe the algorithm with a constant step $\delta>0$ :

$$
\left\{\begin{array}{l}
\widetilde{x}_{k+1}=x_{k}+\delta z_{k}, \\
x_{k+1}=\widetilde{x}_{k+1}-\frac{f\left(\widetilde{x}_{k+1}\right)}{\left\|\nabla f\left(\widetilde{x}_{k+1}\right)\right\|^{2}} \nabla f\left(\widetilde{x}_{k+1}\right), \\
z_{k+1}=\psi\left(x_{k+1}\right) .
\end{array}\right.
$$

We can use backtracking idea to adjust step size for each iteration.

Choose a small $\varepsilon>0$, an initial step $\widehat{\delta}>0$, some $\lambda \epsilon$ $(0,1)$. Let $x_{k}$ and $z_{k}$ be given. Assume $\delta=\widehat{\delta}$.

1. Check the inequality $\left\|\psi\left(x_{k}\right)\right\|>\left\|\psi\left(x_{k}+\delta z_{k}\right)\right\|$

(a) If it is satisfied then build

$$
\left\{\begin{array}{l}
\widetilde{x}_{k+1}=x_{k}+\delta z_{k}, \\
x_{k+1}=\widetilde{x}_{k+1}-\frac{f\left(\widetilde{x}_{k+1}\right)}{\left\|\nabla f\left(\widetilde{x}_{k+1}\right)\right\|^{2}} \nabla f\left(\widetilde{x}_{k+1}\right), \\
z_{k+1}=\psi\left(x_{k+1}\right)
\end{array}\right.
$$

and go to the point 2 of the algorithm.

(b) If it is not satisfied then shrink the step $\delta=\lambda \delta$ and go to the point 1 of the algorithm.

2. Check the stopping criteria $\left\|\psi\left(x_{k+1}\right)\right\| \leq \varepsilon$.

(a) If it is true take $x_{k+1}$ as the solution and finish computations.

(b) If it is not true take $\delta=\widehat{\delta}$, increase $k$ by one and go to the point 1 of the algorithm.

It should be noted that reduction of $\delta$ stops at some moment since $z_{k}$ is a descent direction for the function $\|\psi(x)\|$ at the point $x_{k}$.

Note that modifications of the algorithm with velocity zeroing at each iteration are first order methods, while an algorithm with zero-mass is a second order method.

\section{Numerical experiments}

In this section the results of numerical experiments are given for the problem of finding the minimum distance from the origin to ellipsoids, whose semi-axes are parallel to the coordinate axes and are generated using a generator of numbers uniformly distributed on a segment from 2 to 5 . The centers of the ellipsoids are obtained using a random variable generator, uniformly distributed on the sphere of radius 5 centered at the origin.

Thus we consider the problem of orthogonal projection of the origin onto an ellipsoid

$$
\Omega=\left\{x \mid(x-c)^{T} A(x-c) \leq 1\right\},
$$

where $A$ is a diagonal $n \times n$ matrix with diagonal elements from segment $(2,5)$ and $c$ lies on the sphere of radius 5 centered at the origin.

For each of the dimensions 2, 3, 10, 100, 500, 1000 one hundred test problems are solved and the average calculation time is displayed. In all cases condition (4) is selected as the stopping criteria. The following notations are used:

- CBM - the original Charged Ball Method (3);

- CBM_m - zero-mass modification of the Charged Ball Method (6);

- CBM_v - speed zeroing modification of the Charged Ball Method with a constant step (7);

- CBM_vf - speed zeroing modification of the Charged Ball Method with based on backtracking idea;

- PM - exterior penalty function method using the Newton's Method to solve unconstrained optimization problem at each iteration (see for example [24]); 
- BM - fast geometric method developed and proposed in [5] for solving the problem of projection of a point onto a set.

- EM: ellipsoid method (for example see [6]).

Computations were made in MatLab. The following parameters were chosen for the methods:

- CBM: $p_{1}=30, p_{2}=1, \delta=0.7$;

- CBM_m: $p_{1}=10, p_{2}=1, \delta=0.8$;

- CBM_v: $\delta=8$;

- CBM_vf: $\lambda=50, \delta=50$;

- PM: the penalty factor $r=1000$ increases 10 times at each iteration;

For each method we tried to choose the best parameters possible. We studied many variants and present here the best obtained time of computations for every considered algorithm.

For the dimensions more than 10 Ellipsoid Method works too long, therefore this data is not presented in Table 2. Figure 1 shows a graphic illustration of the obtained results.

Table 1: The average time in seconds for the problems solution with various dimensions dim.

\begin{tabular}{llll}
\hline $\operatorname{dim}$ & CBM & CBM_m & CBM_v \\
\hline 2 & $12.7 \cdot 10^{-5}$ & $12.4 \cdot 10^{-5}$ & $4.1 \cdot 10^{-5}$ \\
3 & $9.8 \cdot 10^{-5}$ & $13.2 \cdot 10^{-5}$ & $3 \cdot 10^{-5}$ \\
10 & $10.6 \cdot 10^{-5}$ & $18.2 \cdot 10^{-5}$ & $3.4 \cdot 10^{-5}$ \\
100 & 0.00227 & 0.00528 & 0.00065 \\
500 & 0.13066 & 0.20174 & 0.02686 \\
1000 & 0.71287 & 1.0857 & 0.10403 \\
\hline
\end{tabular}

Table 2: The average time in seconds for the problems solution with various dimensions dim.

\begin{tabular}{llllll}
\hline $\operatorname{dim}$ & CBM_vf & PM & BM & EM \\
\hline 2 & $30 \cdot 10^{-5}$ & 23.8 & $\cdot$ & 10.8 & 33.2 \\
& & $10^{-5}$ & $10^{-5}$ & $10^{-5}$ \\
3 & 19.8 & 25.2 & $\cdot$ & $8.1 \cdot 10^{-5}$ & 47.9 \\
& $10^{-5}$ & $10^{-5}$ & & & $10^{-5}$ \\
10 & $12 \cdot 10^{-5}$ & 30.6 &. & $9.4 \cdot 10^{-5}$ & 531.3 \\
& & $10^{-5}$ & & $10^{-5}$ \\
100 & 0.00211 & 0.00654 & 0.00204 & - \\
500 & 0.0923 & 0.19271 & 0.06949 & - \\
1000 & 0.35232 & 0.75328 & 0.2993 & - \\
\hline
\end{tabular}

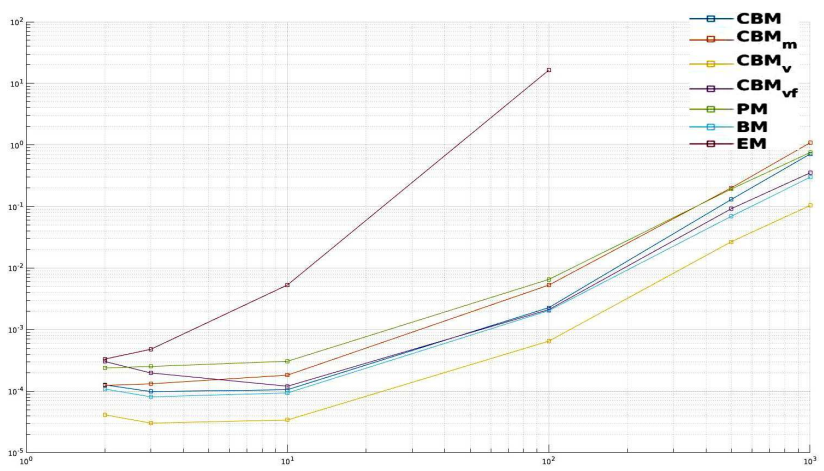

Figure 1: Relation between the average calculation time and the dimension of the problem for the studied methods.

\section{Conclusion}

We can conclude that the method CBM_v is the fastest. The EM method is the worst.

The remaining methods show comparable calculations time. We can mark only the method CBM_m from this group, which works more slowly and shows results comparable to PM on dimensions greater than 100 . This is explained by the fact that both algorithms use the Newton method at each iteration. Therefore they require quite complex (for large dimensions) calculations related to the inversion of Hessian matrices.

Acknowledgement: The reported study was supported by Russian Science Foundation, research project No. 18-7100006.

\section{References}

[1] Abbasov M. E., Charged ball method for solving some computational geometry problems, Vestnik St. Petersburg University, Mathematics, 2017, 50(3), 209-216

[2] Minu M., Mathematical Programming. Theory and Algorithms [in Russian], Nauka, Moscow, 1990

[3] Nocedal J., Wright S., Numerical Optimization, Springer-Verlag New York, 2006

[4] Kochenderfer Mykel J., Wheeler Tim A., Algorithms for Optimization, The MIT Press, Massachusetts, 2019

[5] Lin A., Han S. P., On the distance between two ellipsoids, SIAM J. Optim., 2002, 13, 298-308

[6] Bland R. G., Goldfarb D., Todd M. J., The ellipsoid method: A survey, Operations Research, 1981, 29(6), 1039-1091 